\title{
Feature Extraction and Redesign of Bronze Geometry patterns in Shang and Zhou Dynasties of China
}

\author{
Hua Qi Yao \\ College of Biomass Science and Engineering \\ Sichuan University \\ Chengdu, China
}

\author{
Zhang Gao Peng \\ College of Biomass Science and Engineering \\ Sichuan University \\ Chengdu, China
}

\begin{abstract}
The geometric patterns of bronze in the Shang and Zhou dynasties of China were simple but meaningful which had extremely high practical value and artistic value. However, simple extraction and application can no longer meet the aesthetic needs of contemporary people. Based on the collection and analysis of the textual and picture data in the Shang and Zhou dynasties, the geometric patterns of bronze are extracted by the method of archeological typology, redesigned by combining with the shape grammar, and finally, the innovative patterns are applied to the design of silk scarves. The redesigned geometric patterns of the Shang and Zhou dynasties inherit the unique style and cultural connotation of the geometric patterns, and at the same time, they have the aesthetic feeling of simple and fashionable design of modern patterns. The integration of traditional and modern design can effectively enhance the cultural connotation of products, and promote the inheritance and development of China's excellent civilization.
\end{abstract}

Keywords-Shang and Zhou dynasties dynasties;geometric pattern; ; shape grammar;Redesign;

\section{INTRODUCTION}

There are many kinds of bronzes with various shapes and forms in Shang and Zhou dynasties which patterns on the surface are also unique.Until today, artists around the world have regarded them as the essence of ancient oriental art. As one of the decoration of bronzes, geometric patterns plays an important role.The famous semiotician Cassirer believes that "symbolized thinking is the most representative feature in human life." These geometric patterns show the spiritual belief of the Chinese nation with the most intuitive image in the long process of cultural inheritance. Applying this "meaningful form" in modern product design has practical significance for the spread of China's outstanding national culture. However, with the development of the times and the migration of aesthetic consciousness, even the most creative and decorative geometric patterns at that time can no longer meet the spiritual and practical needs of modern people. The current research work focuses on the classification [1], cultural connotation[2], and application, the research on feature extraction and redesign of its classic patterns is still very scarce. How to better inherit and carry forward the imagery style of the bronze geometric ornaments of the Shang and Zhou dynasties is a problem we are currently facing.

This article takes the geometric patterns of the Shang and Zhou Dynasties as the research object. Through the analysis and combing of texts and pictures available in the Shang and
Zhou Dynasties, the archeological typology method is applied to the geometric ornaments with obvious morphological characteristics and deep cultural connotations in the Shang and Zhou Dynasties.using the shape grammar theory for pattern redesign, and in the end the application of the redesign pattern to the silk pattern, realized the mutual verification of theory and practice.

\section{FEATURE ANALYSIS AND EXTRACTION OF BRONZE GEOMETRIC PATTERNS IN SHANG AND ZHOU DYNASTIES}

\section{A. Analysis of artistic characteristics}

The redesign of geometric patterns in the Shang and Zhou dynasties must first summarize and sort out the information about the generation, development, organization, and cultural meaning of the patterns, which will not only help to clarify the deep genes behind the patterns, but also extract the useful pattern features more accurately, and lay a good foundation for the pattern redesign later.

During the Shang and Zhou dynasties, from the Erligang culture period to the early spring and Autumn period, the development of geometric patterns on bronzes can be divided into three important periods.(i)Erligang culture period. Several forms of quaint geometric patterns have appeared, such as circle patterns (also known as continuous pearl patterns), circular swirl patterns, rhombus patterns, string patterns, cloud and thunder patterns and so on. Among them, the cloud and thunder pattern is one of the geometric patterns with the highest frequency and the most variety in Shang and Zhou bronzes. In this period, the form of geometric patterns is simple, and the style is rough, which belongs to the budding and nascent period of bronze ornamentation.(ii)From the middle Shang Dynasty to the early Western Zhou Dynasty. In this stage, the pattern is represented by the "three layers of flowers" composed of the main pattern and the cloud and thunder pattern. The pattern is rich and gorgeous. At the same time, the geometric patterns formed by the simplification of animal patterns and early varieties of geometric patterns appeared, such as vertical (inverted) triangle pattern, vertical leaf triangle pattern, etc., and began to trend towards geometry.(iii)Mid Western Zhou Dynasty to early Spring and Autumn. At this stage, the geometric pattern became the main decorative pattern, and the mysticism on the pattern was greatly weakened. The decoration has changed from bustling and delicate to simple and elegant. Among them, geometric patterns such as tile pattern, and ring-shaped patterns and 
double-ring patterns that have evolved from the simple animal patterns are the most popular.

From the pattern organizational, the organization pattern of the Shang and Zhou Dynasties's bronze represented the unique aesthetic and religious concepts at that time. Through the organization of the patterns to achieve certain psychological effects and high artistic achievements. There are three main ways (1) Symmetrical. The shape and position of the pattern are mirrored with the straight line as the axis. The layout of the decoration is balanced, stable and solemn; (2) The primary and secondary are distinct. The position and area of the primary and secondary patterns are significantly different, achieving a prominent theme pattern. At the same time, the auxiliary patterns can play a supporting role, giving people a sense of hierarchy, rhythm and order. (3) Continuous pattern. A pattern unit is continuously extended to the left or right or all around to form a continuous pattern.In addition, a single pattern composed of two-direction continuous, two to three kinds of patterns are often arranged alternately at intervals, and the four-direction continuous is common in the Western Zhou Dynasty.

Judging from the implication of the patterns, the geometric pattern is different from the animal pattern tin he Shang and Zhou Dynasties. It accumulates rich social content, religious beliefs and national spirit, and is a "meaningful form", which shows the overall development of early art towards rationalization trend.For example, cloud and thunder patterns abstracted from natural forces such as clouds and thunder which represent praying for auspicious peace and worship of nature. These patterns express the original ancestors , attempts to use abstraction to transcend the way of thinking. After historical sedimentation and cultural baptism, they are rich in cultural connotations and philosophical ideas, and are the crystallization of the wisdom of our ancestors.

\section{B. Geometric pattern feature extraction}

The similar characteristics were classified through sorting and collecting the paper and picture data of the Shang and Zhou dynasties' s bronze geometric patterns. The typical characteristics of cloud and thunder patterns, four petal patterns, triangle patterns, circle patterns, vortex patterns, as well as the Qiequ patterns, ring patterns and Ribbon pattern, which were widely popular in the Western Zhou Dynasty, were selected as the generation of the geometric patterns of Shang and Zhou dynasties.Table 1 summarizes the pattern organization, pattern implication, as shown below.

TABLEI 1 Analysis of Shang and Zhou Geometric Pattern Features

\begin{tabular}{|c|c|l|l|}
\hline Name & Physical copy & $\begin{array}{c}\text { Pattern } \\
\text { organization }\end{array}$ & \multicolumn{1}{c|}{$\begin{array}{c}\text { Pattern } \\
\text { Meaning }\end{array}$} \\
\hline Cloud pattern & Symmetrical & $\begin{array}{l}\text { Auspicious } \\
\text { Wealthy }\end{array}$ \\
\hline Thunder pattern & Continuous & $\begin{array}{l}\text { Symmetrical } \\
\text { Continuous }\end{array}$ & $\begin{array}{l}\text { Auspicious } \\
\text { Wealthy }\end{array}$ \\
\hline Four petal patterns & Symmetrical & $\begin{array}{l}\text { Natural } \\
\text { Harmony }\end{array}$ \\
\hline Triangle patterns & Alternation & Continuous \\
\hline Circle patterns & nature worship \\
\hline Vortex pattern & Continus & Eternal \\
\hline
\end{tabular}

\begin{tabular}{|c|c|c|c|}
\hline Qiequ patterns & (9) (9) & Continuous & Ancestor worship \\
\hline Ring patterns & 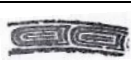 & Continuous & Ancestor worship \\
\hline Ribbon pattern & 19:\% & Continuous & Ancestor worship \\
\hline
\end{tabular}

No matter how the pattern change, it always has its core characteristics. This article uses archeological typology to compare and study the process of pattern morphology change, to make a comparative study of similar patterns of different types, to find out the pattern of geometric pattern evolution. The in-depth investigation of the typical Shang and Zhou bronze geometric patterns in Table 1 is divided into three types: I, II, and III. Each type represents the most popular and important style at that time. By comparing the styles popular in different periods to find out the evolution of the pattern and extract the core features.For example, by comparing the three types of cloud thunder patterns in different periods, we can find that the core feature is that the lines are rounded with "rounded corners" to give people a gentle sense, while the thunder patterns are "squared corners" to give them a strong sense of rigidity; in this way, the features of each pattern are extracted as shown in Table 2.

TABLE 2 Evolution and feature extraction of geometric patterns

\begin{tabular}{|c|c|c|c|c|}
\hline Name & $I$ & II & III & $\begin{array}{l}\text { Feature } \\
\text { extraction }\end{array}$ \\
\hline $\begin{array}{l}\text { Cloud } \\
\text { pattern }\end{array}$ & 瓷党 & 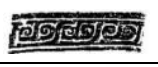 & 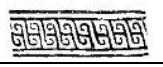 & ()(2) \\
\hline $\begin{array}{l}\text { Thunder } \\
\text { pattern }\end{array}$ & 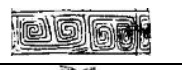 & (6) & magenen & \begin{tabular}{|l|}
$\square$ \\
\end{tabular} \\
\hline $\begin{array}{c}\text { Four petal } \\
\text { patterns }\end{array}$ & 36s & & & \\
\hline $\begin{array}{l}\text { triangle } \\
\text { patterns }\end{array}$ & 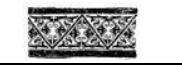 & & $\sqrt{15}$ & \\
\hline $\begin{array}{c}\text { Circle } \\
\text { patterns }\end{array}$ & 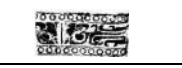 & & & \\
\hline $\begin{array}{l}\text { Vortex } \\
\text { pattern }\end{array}$ & (2) & & & \\
\hline $\begin{array}{c}\text { Qiequ } \\
\text { patterns }\end{array}$ & 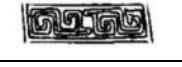 & 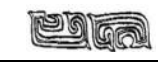 & 6) & \\
\hline $\begin{array}{c}\text { Ring } \\
\text { patterns }\end{array}$ & Nyvay & 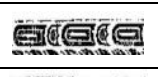 & $\mathrm{B}$ & \\
\hline $\begin{array}{l}\text { Ribbon } \\
\text { pattern }\end{array}$ & คㅇำ & 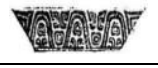 & 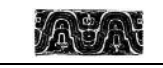 & \\
\hline
\end{tabular}

\section{REDESIGN OF GEOMETRIC PATTERNS}

\section{A. Shape grammar}

Shape grammar (SG) is a design method composed of shapes and deduction rules. This method generates a new pattern that can retains initial shape style continuity according to the established deduction rules, and also generates a large number of different design schemes for designer. Shape grammar was first proposed by George Stiny and Gips, and applied it to painting and sculpture design[3], and later extended to architectural design[4], product design[5], creative design[6], brand design[7], and other design fields.

According to the definition of Stiny and Gips, the shape grammar can be expressed as a quaternion: $\mathrm{SG}=(\mathrm{S}, \mathrm{L}, \mathrm{R}, \mathrm{I})$ : $\mathrm{S}$ is a finite set of shapes; $\mathrm{L}$ is a finite set of labels; $\mathrm{R}$ is a regular Finite set; I is labeled initial shape. The shape rule $\mathrm{R}$ defines the evolution of existing shapes; SG is a set of shapes generated by $\mathrm{S}$ through regular rule operations such as translation, rotation, mirroring, and scaling. Marks are used to explain that transformation rules are applied to specific parts 
of the image. Define the deduction rules R1 is horizontal movement; R2 is tilt movement; R3 is proportional scaling; $\mathrm{R} 4$ is horizontal mirror; R5 is vertical mirror; R6 is rotated 45 around the bottom, $\mathrm{R} 7$ is rotated $45^{\circ}$ around the center; R8 is rotated around the bottom $90^{\circ}$, the essence of its shape transformation is to change the coordinates of each vertex of the two-dimensional space graphics by transforming the matrix. Taking the "Four petal patterns" as an example, the effect of the evolution rules of shape grammar is shown in Table 3.

\section{B. Redesign experiment of geometric patterns}

Based on the above definition of shape grammar, once the geometric pattern features of Shang and Zhou dynasties are effectively extracted as the initial shape of the grammar operation mechanism, the pattern can be redesigned by controlling (1) the initial shape (2) the deduction rules (3) the deduction sequence. In the first stage, the extracted geometric pattern factors of the Shang and Zhou dynasties are generated and deduced. The design scheme is shown as follows.

TABLE 3 Evolution rules of shape grammar patterns

\begin{tabular}{|c|c|c|c|c|}
\hline $\begin{array}{c}\text { Rule } \\
\text { Name }\end{array}$ & $\begin{array}{c}\text { horizontal } \\
\text { movement }\end{array}$ & $\begin{array}{c}\text { Tilt } \\
\text { movement }\end{array}$ & $\begin{array}{c}\text { Proportional } \\
\text { scaling }\end{array}$ & $\begin{array}{c}\text { horizontal } \\
\text { mirror }\end{array}$ \\
\hline legend & & & & \\
\hline $\begin{array}{c}\text { Rule } \\
\text { Name }\end{array}$ & $\begin{array}{c}\text { Vertical } \\
\text { mirror }\end{array}$ & Rotated $45^{\circ}$ & Rotated $45^{\circ}$ & Rotated $90^{\circ}$ \\
\hline legend & 5 & &
\end{tabular}

Evolution scheme one: Keep the deduction rules and deduction order unchanged, set the number of deductions $\mathrm{N}=$ 7, and innovate and evolve the Shang and Zhou geometric patterns by introducing different initial shapes. Apply the derivation rule R6 sequentially: rotate $45^{\circ}$ around the bottom as an example, and introduce triangle patterns, circle patterns and thunder patterns with different initial shapes for pattern evolution. The evolution results are shown in Fig. 1

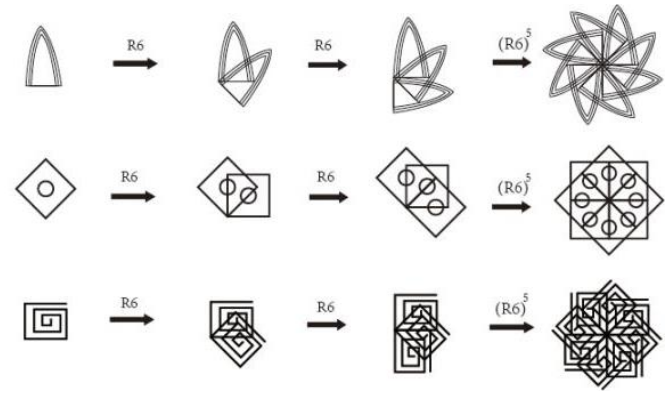

Fig.1Evolution scheme one

Evolution scheme two: Keep the original shape and deduction order unchanged, and change the deduction rules to carry out innovative evolution of geometric patterns. Evolution experiment one: Introduce lightning patterns as the initial shape, control the inversion order to be alternating, the number of inferences is $\mathrm{N}=3$, and set the inference rules to R4: horizontal mirror image, R5 vertical mirror image, to perform pattern evolution; evolution experiment two, introduce lightning pattern as the initial shape., Control the deduction sequence to be alternating, the number of deductions $\mathrm{N}=3$, set the deduction rule $\mathrm{R} 2$ : tilt movement, deduction rule R5: vertical mirroring, to carry out pattern evolution; evolution experiment three, introduce the thunder pattern as the initial shape, control the deduction sequence to be alternate, and set the derivation rules R1: horizontal movement, R5: vertical mirroring, and pattern evolution. The evolution results are shown in Fig. 2

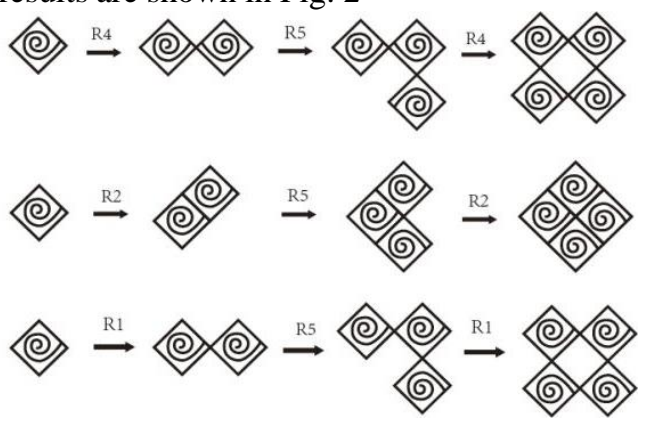

Fig. 2 Evolution scheme two

Evolution scheme three: Keep the initial shape and deduction rules unchanged, the geometric pattern innovation evolves by changing the deduction order. The circle pattern is introduced as the initial pattern, and the deduction rule is set to R2 tilting movement; R4 is horizontally mirrored, the number of deductions is $\mathrm{N}=3$, the deduction order is changed, and the pattern evolution result is shown in Fig. 3

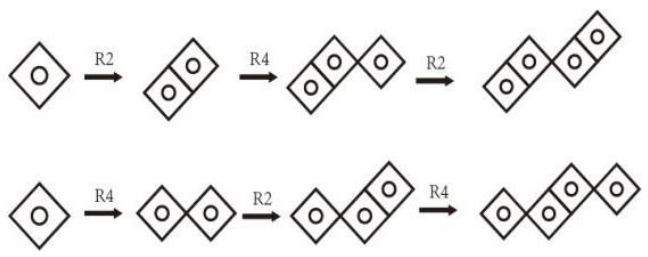

Fig. 3 Evolution scheme three

Through the above three different evolution schemes to conduct geometric pattern deduction experiments, a series of derived geometric patterns were obtained, as shown in Table 4.

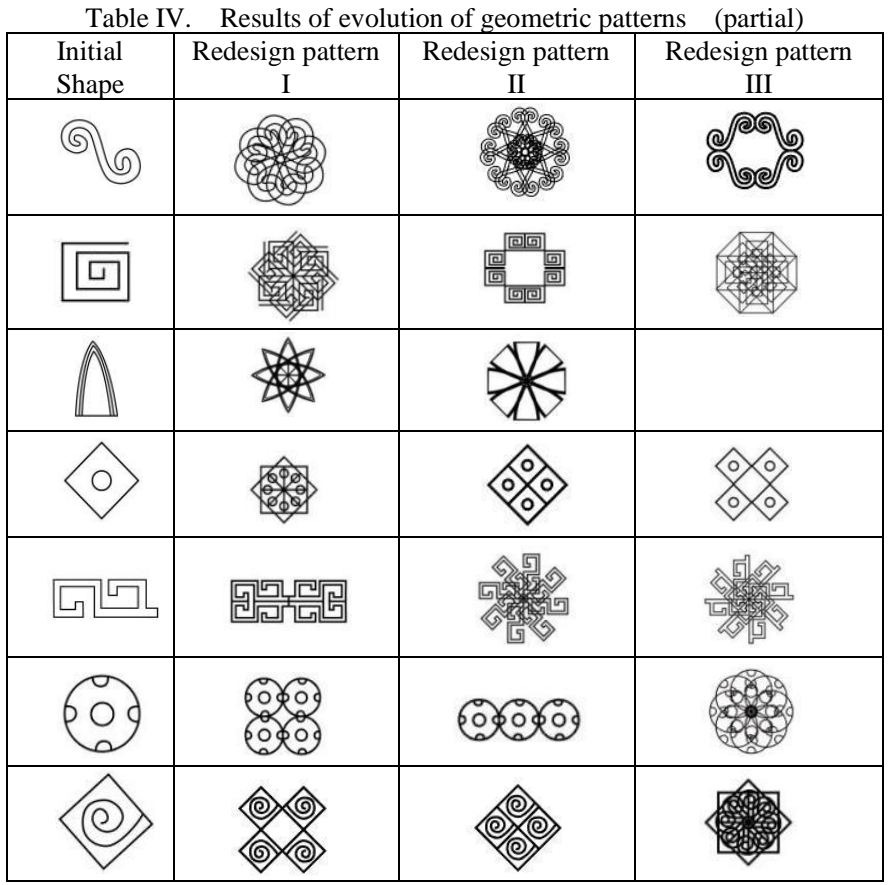




\begin{tabular}{|c|c|c|c|}
\hline$\square$ & 吕呵 & 要 & 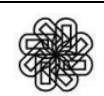 \\
\hline & 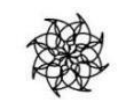 & (2) & हैy \\
\hline
\end{tabular}

The experimental results show that in the process of designing and applying the shape grammar mechanism: (1) the rules of shape derivation can be repeatedly used alternately, and the translation of the shape can also achieve scaling, rotation and other changes. (2) Under the condition that the derivation rules and derivation order are not changed, the patterns derived from different initial shapes using the same rules are completely different. (3) Under the condition that the initial shape and the inference order are not changed, different or similar patterns can be obtained by applying different inference rules. (4) Under the condition that the initial shape and the derivation rules are unchanged, the order in which the derivation rules are used is different, and the resulting patterns will be different. By comparing and analyzing the features of the initial pattern and the features of the evolved pattern, it can be seen that the new patterns generated by generative inherit the basic features and visual style of the initial pattern. While generating new features, the symbolic meaning of the initial pattern is well retained To lay the foundation for the next stage of derivative evolution.

In the second stage, the basic figures obtained from the first evolution result are regarded as a whole, and the process of further application of the deduction rules is called derivative deduction, which will result in more complex and changeable geometric patterns. The pattern generated by the evolution of thunder pattern is introduced into the grammar mechanism as the initial shape, and the deduction rule $\mathrm{R} 8$ is applied to rotate $90^{\circ}$ around the point. Further shape deduction is carried out, and the pattern effect is shown in Figure 4. Take the patterns generated by the evolution of cloud patterns as a whole, perform R2 tilt movement twice, R4 horizontal mirror once, R5 vertical mirror once and R4 horizontal mirror once respectively to get new patterns.

\section{GEOMETRIC PATTERN REDESIGN AND APPLICATION}

The pattern is composed of pattern unit, composition and color. The pattern unit is the basis of the pattern. At present, the feasibility of generating a large number of innovative patterns while inheriting the traditional style visual style for the shape grammar has been confirmed by multi-field and multi-level practice. However, there are few compositional designs for evolution patterns. The essence of the beauty of patterns is the perfect combination of content and form. Therefore, the design of patterns necessarily requires a structural and systematic concept. This section focuses on the composition research of the geometric pattern of Shang and Zhou Dynasties generated based on shape grammar as the basic unit of the pattern. Using a $52 \mathrm{~cm} \times 52 \mathrm{~cm}$ square silk as the pattern carrier, the evolved commercial and Zhou geometric patterns are patterned according to three different composition methods.

1) Symmetric composition. Symmetry is a classic form of silk composition. The pattern organization is characterized by a neat and uniform combination relationship. The symmetrical pattern of the structure gives people a sense of solemnity, stability, cleanliness and harmony. The theme is represented by auspicious cloud and thunder patterns, supplemented with triangle patterns to decorate the silk pattern. The colors are selected from the traditional Chinese colors of cyan and goose yellow, and supplemented by pine green, turquoise and begonia red. It is stylish, lively, noble, restrained, and at the same time, without losing the national style. The effect is shown in Fig. 5
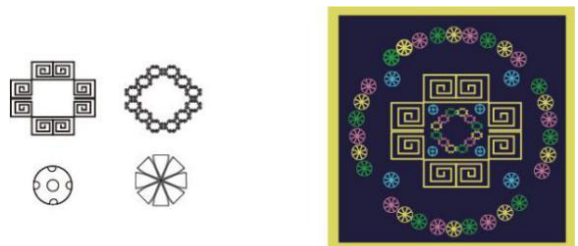

Fig.5 Symmetrical silk pattern design

2)Contrast composition. The contrast form is also one of the more commonly used in the design of silk. Usually, the pattern elements of different shapes, colors, sizes and directions are juxtaposed, such as curves and lines, large and small, warm and cold, thick and thin, soft With hard, deep and shallow, it forms a different and individual pattern effect, which enhances the artistic appeal of the pattern. When using the contrast form, it is often necessary to use a reconciliation method to achieve the beauty of both contrast and harmony, which makes the pattern clear and affirmative.The silk design is based on the motif design series and circular swirl pattern, which represents auspicious and richness. The color match uses a lower purity silver red as the background color, supplemented by cypress green, cyan and goose yellow for warm and cold contrast. Visually, The pattern is the extraction, simplification and combination of Shang and Zhou geometric patterns, which has considerable recognition and cultural appeal, as shown in Fig. 6.

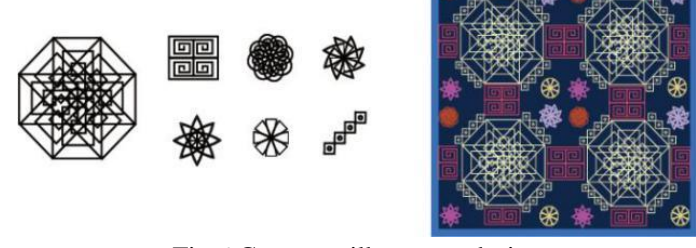

Fig.6 Contrast silk pattern design

3)Repeated composition. The basic patterns are repeatedly repeated in the same or similar manner, alternately combined, and arranged to produce various patterns. The patterns can be repeated regularly, or they can be arranged to gradually change. Regular repetition gives people a sense of stability, uniformity, and gradual repetition gives people a sense of denseness and rhythm. As shown in Figure 7, the silk pattern design is based on the theme of the series of cloud pattern triangle pattern and circle pattern. The main pattern and the auxiliary pattern are continuously developed in four directions, and the low-saturation warm and cold contrast is used for color matching. , Tidy and unified, giving a calm feeling, the effect is shown in Fig .7 

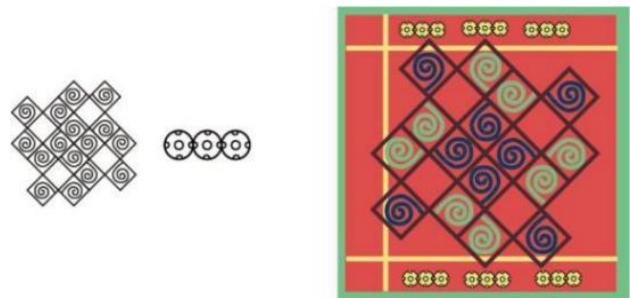

Fig.7 Repeated composition silk design

\section{CONCLUSION}

Tradition is not the opposite of modern, but the source of modernity. A systematic, scientific and effective method can greatly enhance the spiritual and cultural connotation of modern product design, and at the same time promote the spread and development of China's excellent culture. In this paper, the characteristics of the Shang and Zhou bronze geometric patterns with aesthetic value and cultural connotation were extracted by inductive summarization, mutual verification of graphics and texts, and archeological typology. Based on this, modern computer graphics were introduced. The shape method innovates and evolves the inference rules between the initial shape, the inference order and the inference rules. On top of this, three kinds of composition design researches are carried out on the evolved geometric patterns with silk scarves as carriers, and good results are obtained. It provides research ideas for solving the inheritance and innovation of bronze geometric patterns in Shang and Zhou Dynasties, improving the innovation of current geometric patterns and the cultural nature of product design. In addition, this research framework has strong robustness, and it is also useful for the design of other patterns. It has certain reference value.

\section{REFERENCES}

[1] Ni Yuzhan."Interactive Relationship between Technology and Art in Bronze Vessels Design of Xia,Shang and Zhou Dynasties", Decoration, Issue 271,November 2015.pp:85-87.

[2] YU Senlin,TANG Minyuan,HAN Min."On the Horrific Experience of Bronze Decoration of the Childlike Beauty",Packaging Engineering,Vol. 38, Issue 10,May 2017.pp:214-217.

[3] GeorgeStiny."Introduction to shape and shape grmmars", Environment and PlanningB,Vol.7,July 1980,pp. 343-351.

[4] .Buthayna Eilouti."Shape grammars as a reverse engineering method for the morphogenesis of architectural facade design",Frontiers of Architectural Research,Vol.39,Issue 2,August 2019,pp:191-200.

[5] LuZhaolin,TangWencheng,XueCheng,"Method of design DNA reasoning based onshape grammar",Journal of Southeast University,china,Vol.40,Issue4,July 2010.pp:705-711.

[6] Cui Jia, Tang Ming-xi." Integrating Shape Grammars into a Generative System for Zhuang Ethnic Embroidery Design Exploration", Computer-Aided Design, ,Vol.45,Issue3, March 2013, pp: 591-604.

[7] PuglieseM,CanganJ."Capturing aRebel: Modeling The Harley-Davidson Brand Through a Motorcycle Shape Grammar.",Research in Engineering Design, Vol.13,April 2002, pp: $139-156$. 